\title{
Metal-Only Transmitarray Based on C-Shaped Slot
}

\author{
Kien Pham ${ }^{1,2}$, Ronan Sauleau ${ }^{1,2}$, Erwan Fourn ${ }^{1,3}$ \\ ${ }^{1}$ IETR, UMR CNRS 6164, France \\ ${ }^{2}$ Université de Rennes 1, 35042 Rennes Cedex, France \\ ${ }^{3}$ INSA de Rennes, CS70839, 35708 Rennes Cedex \\ trung-kien.pham.1@univ-rennes1.fr, ronan.sauleau@univ- \\ rennes1.fr, erwan.fourn@insa-rennes.fr
}

\begin{abstract}
This paper presents a novel design of metal-only transmitarray using unit-cells based on C-shaped slots. The unitcell consists of four identical layers separated by a quarterwavelength spacer at $20 \mathrm{GHz}(3.75 \mathrm{~mm})$. The phase variation reaches $360^{\circ}$ by varying the inner radius of the slot. An example of linearly-polarized transmitarray is provided to validate this concept at K-band for satellite communications in the down-link band.
\end{abstract}

Keywords - metal-only transmitarray, Ka-band, Satcom

\section{INTRODUCTION}

Transmitarrays antennas (TAs) are mature and eligible antenna technologies for many applications demanding high gain and relatively wide bandwidth. Thanks to its spatially-fed configuration in transmission mode, TAs do not suffer from feed blockage as observed in reflectors or reflectarray antennas. In addition, TAs exhibit lower insertion loss as compared to phased arrays whose beam forming network is complex and relatively lossy at higher frequency. Many different types of TA prototypes have been demonstrated in the past with high gain (>30 dBi), broad bandwidth, dual-band operation and/or dual-polarization, beam-switching and steering capabilities, etc. Among those, most of the corresponding TA demonstrators are fabricated using printed circuit board (PCB) technology; the associated fabrication cost contributes to a major part of the total cost of the antenna and leads to rather expensive solutions for mass product applications. This motivates a recent interest on a very lowcost solutions based on TAs without dielectric substrates, also referred to as metal-only transmitarray antennas (MOTAs).

Several publications illustrate this trend and have validated such concepts in X-band [1]-[2] and Ku-band [3]-[4]. Our objective is to demonstrate a low-cost transmitarray for ground terminals operating in the Satcom downlink K-band (17.7 $21.2 \mathrm{GHz}$ ). The proposed unit-cell is based on a C-shaped slot frequency selective surface operating as a band pass filter. It is described in Section II. An example of 20-by-20 element TA is designed and its performance is presented in Section III.

\section{UNIT-CELL FOR METAL-ONLY TRANSMITARRAY}

\section{A. Geometry}

The three-dimensional geometry of the proposed unit-cell is depicted in Fig. 1. This cell is based on a C-shaped slot frequency selective surface, with a periodicity of $7 \mathrm{~mm}$, which is less than half of a wavelength at $18.75 \mathrm{GHz}$. The metal layer

This works is supported by the French National Research Agency (ANR) in the project named TRANSMIL (TRANSmitarray antenna at MILlimeterwave) under Grant ANR-14-CE28-0023.

\author{
Fatimata Diaby, Antonio Clemente, Laurent Dussopt \\ CEA, LETI, MINATEC Campus, 38054 Grenoble \\ Université Grenoble-Alpes, 38000 Grenoble \\ Grenoble, France \\ fatimata.diaby@cea.fr, antonio.clemente@cea.fr, \\ laurent.dussopt@cea.fr
}

thickness is $500 \mu \mathrm{m}$, and the material is chosen to be conductive, e.g. copper, aluminum, etc. A four-layer stack-up configuration is selected to cover a phase-shift range of $360^{\circ}$. The layer separation is about quarter wavelength $(4 \mathrm{~mm})$ at $18.75 \mathrm{GHz}$.

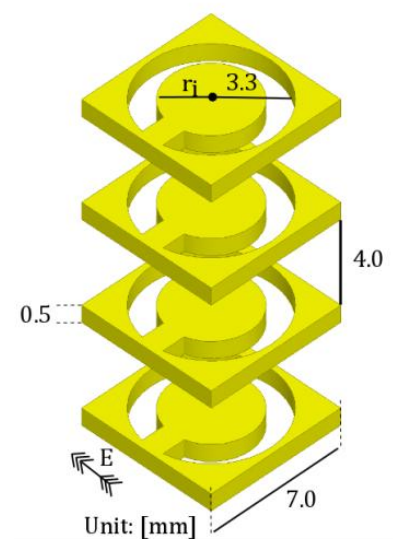

Fig. 1. Geometry of the proposed MOTA unit-cell based on C-shaped slot frequency selective surface. Dimensions are in $\mathrm{mm}$.

The bandpass response of this unit-cell can be shifted by changing the inner radius $r_{i}$ of the $\mathrm{C}$-shaped slot, as shown in Fig. 1. In this study, the outer slot radius is fixed and is equal to $3.3 \mathrm{~mm}$.

\section{B. Performance}

The metal-only unit-cell (MOUC) has been characterized numerically by full-wave simulations using Ansys HFSS v. 15 with periodic boundary conditions. The main results in transmission are presented in Fig. 2 for four frequency points in the down-link band. The unit-cell exhibits a phase-shift of more than $360^{\circ}$ (Fig. 2b) when the value of the inner radius $r_{i}$ varies between $1.5 \mathrm{~mm}$ and $2.7 \mathrm{~mm}$. It is interesting to see that the phase curves are almost parallel; this indicates the wide bandwidth properties of the proposed unit-cell. The transmission magnitude at $19 \mathrm{GHz}$ is better than $-3 \mathrm{~dB}$ and higher degradations are observed at the band edges $(18 \mathrm{GHz}$ and $21 \mathrm{GHz}$ ) (Fig. 2a).

\section{EXAMPLE OF METAL-ONLY TRANSMITARRAY}

The proposed MOUCs have been used to design several MOTA prototypes of various sizes. All prototypes radiate at boresight at K-band. 


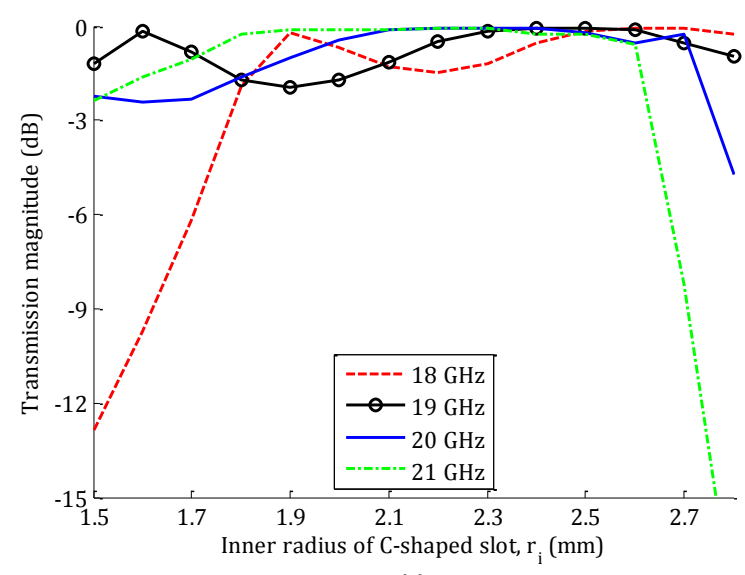

(a)

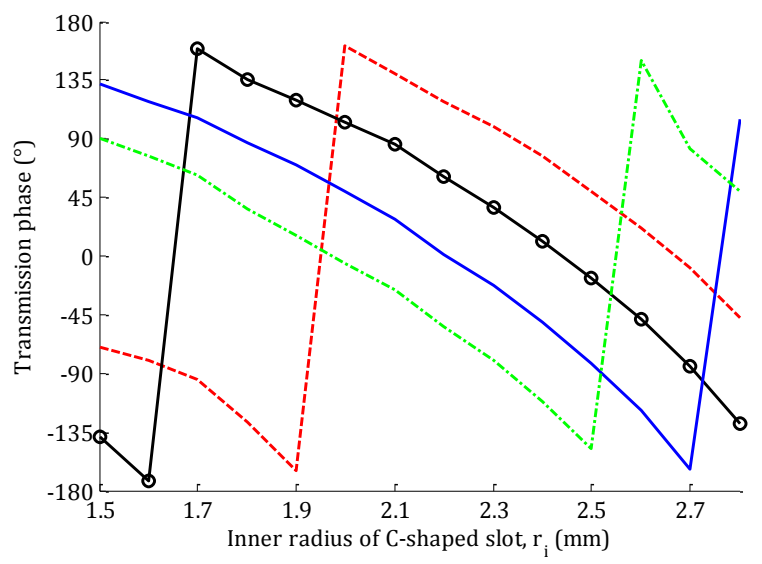

(b)

Fig. 2. Varition of the transmission coefficients in magnitude (a) and phase (b) of the metal-only unit-cell as a function of the inner diameter $r_{i}$, for four frequency points in the down-link band.

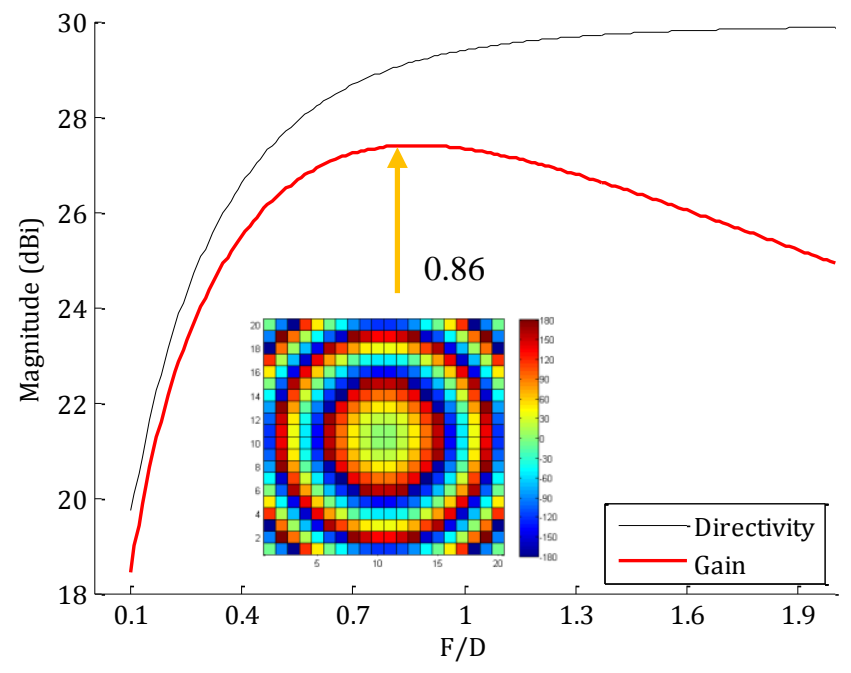

Fig. 3. Computed-directivity and gain of 20-by-20 MOTA for various F/D values at $19 \mathrm{GHz}$. Inset: phase layout for the optimal F/D value (0.86).

We describe here the main performance of one of these prototypes. It has been designed at $19 \mathrm{GHz}$ and contains 400 MOUCs arranged in a 20 -by-20 element square lattice. The directivity and gain are plotted in Fig. 3 as a function of F/D, assuming no insertion loss and ideal phase compensation at 19 GHz. The TA is illuminated by a $10-\mathrm{dBi}$ feed horn. The optimal F/D maximizing the antenna gain is equal to 0.86 , equivalent to a focal length of $122 \mathrm{~mm}$. The computed phase distribution across the transmitarray aperture at $19 \mathrm{GHz}$ is depicted in the inset of Fig. 3 for this optimal position of horn.

The radiation patterns have been computed using array theory [5] and HFSS simulations. The E-plane patterns are represented in Fig. 4. The gain reaches $24 \mathrm{dBi}$ and crosspolarization level below $-40 \mathrm{~dB}$ in all elevation angles.

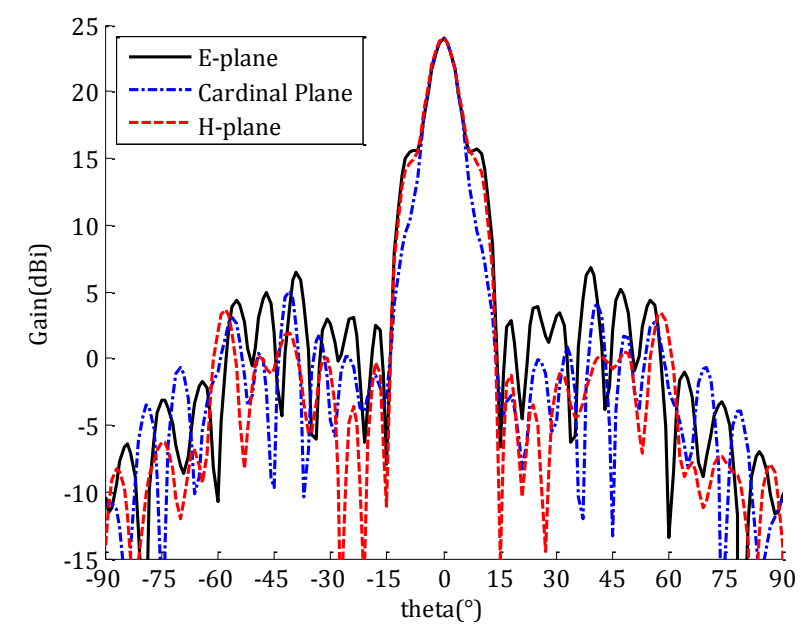

Fig. 4. Simulated co-polarized gain components of the MOTA at $19 \mathrm{GHz}$.

\section{CONCLUSIONS AND PERSPECTIVES}

Metal-only unit-cells and transmitarrays have been introduced for very low cost applications, particularly for Satcom ground terminals at down-link band. The corresponding unit-cells are based on pass-band C-shaped frequency selective surfaces. Fabrication of the prototypes is under progress and detailed experimental results will be presented during the conference (fabrication techniques, assembly, and performance in radiation). The proposed concept can be scaled for other applications (e.g., up-link Satcom in Ka-band, outdoor flat antenna panels for $5 \mathrm{G}$ access points and backhaul links in Ka-band and V-band).

\section{REFERENCES}

[1] A. H. Abdelrahman, A. Z. Elsherbeni, and F. Yang, "Transmitarray antenna design using cross slot elements with no dielectric substrate," IEEE Antennas Wireless Propag. Lett., vol. 13, pp. 177-180, 2014.

[2] B. Rahmati and H. R. Hassani, "High-efficient wideband slot transmitarray antenna," IEEE Trans. Antennas Propag., vol. 63, no. 11, pp. 5149-5155, Nov. 2015.

[3] G. Liu, H. Wang, J. Jiang, F. Xue, and M. Yi, "A high efficiency transmitarray antenna using double split rings slot elements," IEEE Antennas Wireless Propag. Lett., vol. 14, pp. 1415-1418, 2015.

[4] R. Y. Wu, Y. B. Li, W. Wu, C. B. Shi, and T. J. Cui, "High-gain dualband transmitarray," IEEE Trans. Antennas Propag., vol. 65, no. 17, pp. 3481-3488, Jul. 2017.

[5] K. T. Pham, R. Sauleau, E. Fourn, F. Diaby, A. Clemente and L. Dussopt, "Dual-band transmitarrays with dual-linear polarization at Kaband," IEEE Trans. Antennas Propag., vol. 65, no. 12, pp. 7009-7018, Dec. 2017. 